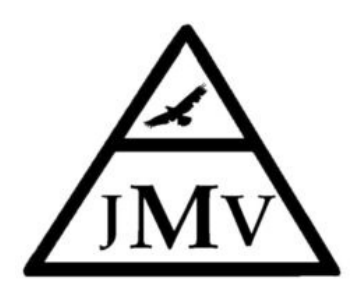

\title{
Electrical Impedance Tomography: the future of mechanical ventilation?
}

\author{
Melina Simonpietri ${ }^{1}$, Mia Shokry ${ }^{2}$, Ehab Daoud MD ${ }^{3}$ \\ DOI: https://doi.org/10.53097/JMV.10024 \\ Cite: Simonpietri M, Shokry M, Daoud EG. Electrical Impedance Tomography: the future of mechanical \\ ventilation? J Mech Vent 2021; 2(2):64-70.
}

\begin{abstract}
:
Electrical Impedance Tomography is a rapidly evolving technology used for bedside lung imaging. Although EIT devices have been commercially available for the past decade, they are still not commonly used in everyday clinical practice. EIT has multiple benefits over standard chest imaging techniques; it is non-invasive, it can be used at bedside and it allows continuous monitoring of the patient's condition.

EIT can change the standard practice for monitoring lung function and caring for patients on mechanical ventilation.

In this concise review, we will discuss the general concepts of EIT and its clinical applications. As this technology keeps developing and becomes more available for clinical use, it might revolutionize the way we practice mechanical ventilation.

Additional studies need to be performed to compare its benefits to our current practice.
\end{abstract}

Keywords: EIT, Mechanical ventilation, PEEP, Overdistention, Ventilator induced lung injury

Authors

1. Melina Simonpietri, Pre-med student, California, USA

2. Shokry Mia, Pre-med student, California, USA

3. Ehab G Daoud, MD, FACP, FCCP. Associated professor of Medicine, John A Burns School of Medicine, Hawaii, USA and director of respiratory care program, Kapiolani Community College, Hawaii, USA

Corresponding author: ehab_daoud@hotmail.com

Conflict of interest/Disclosures: None

Funding: None 


\section{Introduction}

Acute respiratory failure is one of the most common causes of admission to the ICU, it usually requires mechanical ventilation for treatment and results in significant mortality. ${ }^{1}$ Currently, patients that require mechanical ventilation are primarily monitored with clinical examination, blood sampling for oxygenation and ventilation, and data monitoring from the ventilator.

Radiological data is also extensively used for diagnosis and monitoring. Computerized Tomography (CT) scan is considered the gold standard for lung imaging because of its ability to produce images with high resolution. CT has major disadvantages when used for monitoring a patient's condition, it requires moving and transporting a critically ill patient to radiology suites, it exposes the patient to radiation, and it is unable to carry out repeated measures. ${ }^{2}$ Ultrasound (US) has gained momentum over CT scan in the last decade as a less expensive, radiation free alternative that can be used easily and repeatedly at bedside, ${ }^{3}$ however technical training for imaging acquisition is necessary for proper use of this technology.

Electrical impedance tomography (EIT) is an alternative chest imaging method that is easy to use, safe for the patient, and allows continuous bedside monitoring of the patient's condition. Although EIT is a novel technology, it is not commonly used in the United States at the moment. The purpose of this review is to summarize the clinical use and benefits of EIT as an imaging technology.

\section{What is Electrical Impedance Tomography and how does it work?}

Electrical Impedance Tomography (EIT) is a novel noninvasive, radiation free imaging technique that is mainly used for bedside monitoring of the changes in the ventilation and perfusion of lung tissue. EIT is the only bedside lung imaging technology that can be used to assess lung volume changes in real time in a repetitive manner. ${ }^{4}$

EIT devices apply known amounts of low-frequency, high amplitude electric currents through multiple pairs of electrodes (16-32) applied circumferentially to the surface of the thorax. These currents allow the device to determine the conductivity (impedance) of the cross section of the thorax, creating a two- dimensional image of the area under the electrodes. ${ }^{4}$ (Figure 1)

The tissue densities detected is translated to areas of ventilation and consolidation through different regions of both lungs. Additionally, the ability to calculate ventilation gains, losses and change in the end expiratory lung volumes and translate them into a color-coded images. Those regions could be viewed as quadrants or horizontal regions termed regions of interest (ROI).

The images produced by the EIT are presented in a color gradient that varies depending on the machine. The most common is the rainbow scheme, where red represents the high impedance values (liquid), blue represents low impedance values (air), and green represents the baseline. ${ }^{5}$

EIT devices can also be used for functional EIT imaging (fEIT). This type of EIT records the changes in impedance over a period of time and provides useful data to evaluate the homogeneity of ventilation and determine regions of interest (ROIs).

Some animal and human studies ${ }^{6,7}$ have shown a strong correlation between the changes in endexpiratory lung impedance (EELI) and the changes in end-expiratory lung volume (EELV), however, this research is not yet sufficient to estimate EELV based on EELI in clinical practice.

Other useful measures that can be derived from EIT data include the impedance ratio (IR) and the homogeneity index (GI). IR is an index that divides ventilation in the ventral sector of the lungs by the ventilation on the dorsal section of the lung; it proved to be useful for the assessment of recruitment and derecruitment in animal studies. The GI is useful for the assessment of ventilation inhomogeneity between the right lung and the left lung. ${ }^{5}$

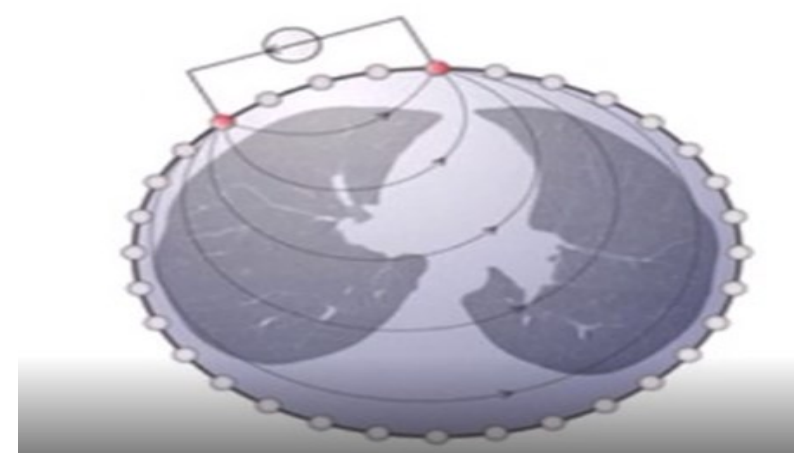

Figure 1: Illustration of 36 electrodes with current generated with one electrode travelling through the thorax and received by another electrode. Courtesy of Timpel reprinted with permission. 


\section{History of Electrical Impedance Tomography}

EIT was first introduced as a geophysical tool for imaging subsurface structures using measurements from the ground surface. In 1978 the mathematical model for geophysical EIT was inverted to fit an EIT device that could be used for medical imaging. Barber and Brown were the first to build a medical EIT device and introduce it as a potential tool in medical imaging. EIT was initially intended to have many different applications including gastric emptying and brain monitoring. Nowadays EIT is mostly used for applications in the lung. The clinical need for monitoring regional lung function at bedside drove the development of the EIT devices used to this date. ${ }^{8}$
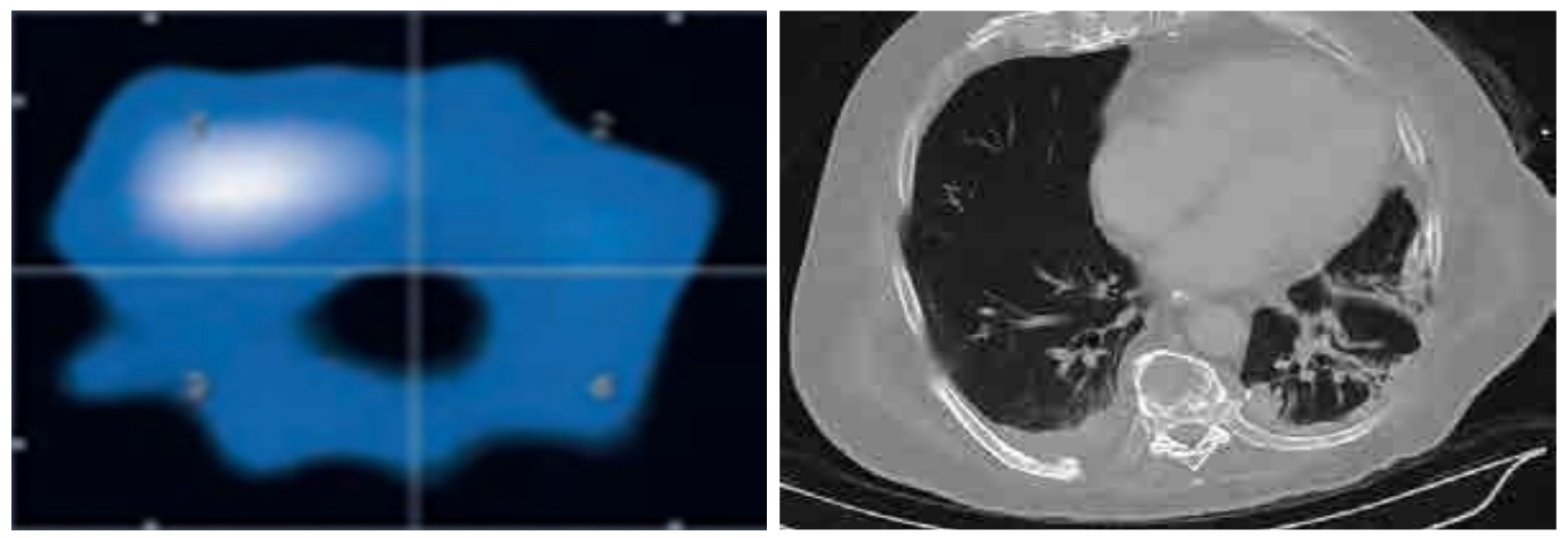

Figure 2: EIT and corresponding CT scan images of ARDS patient showing the marked heterogeneity between dorsal and ventral areas. C) Drager. Image reprinted with permission.
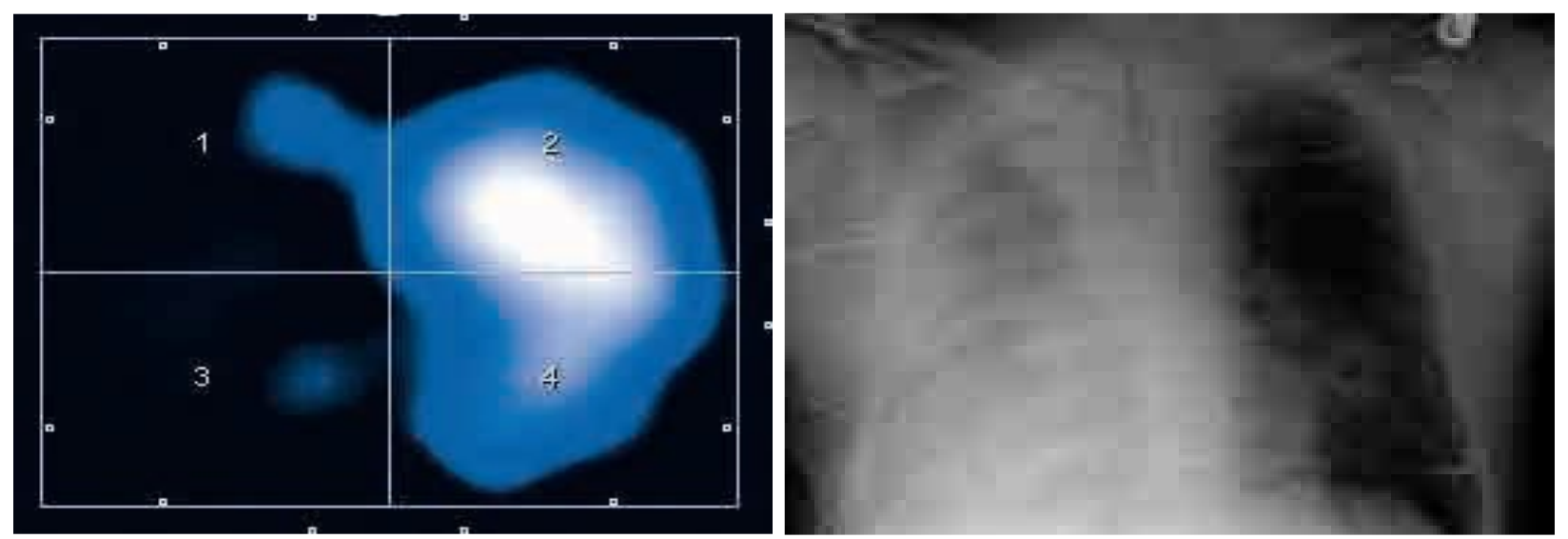

Figure 3: EIT and corresponding chest xray images of patient with right lung ventilation loss (pneumonia, lung collapse, pneumonectomy, etc) (C) Drager. Image reprinted with permission.

\section{Clinical applications:}

Though most of the clinical studies done with EIT are primarily animal or small human series, the multitude of benefits of applying EIT in clinical practice is enormous.

\section{Assessing ventilation inhomogeneity}

Knowledge of ventilation distribution is beneficial in respiratory failure especially in ARDS cases where ventilation is limited in the dorsal areas of lung basis, and in unilateral lung disease. ${ }^{9}$ Figure $2 \& 3$ 


\section{Effect of positioning and Prone}

The effect of gravitational forces and pleural pressure changes play an important role during different patient positions. EIT might help guide best position that improve ventilation and perfusion matching. ${ }^{10}$ This might be especially important during the prone position. ${ }^{11}$

\section{During recruitment maneuvers}

Not every lung especially in ARDS is recruitable. ${ }^{12}$ During the recruitment maneuver, high inspiratory pressure is applied for a brief period of time. ${ }^{13}$

Accurate visual assessment of ventilation gains and hyperventilation during the recruitment maneuver is now visible and calculated instantly.

\section{Setting PEEP}

There is no agreement on what the best method of setting PEEP. ${ }^{14}$ Additionally, no method has shown superiority over other methods. ${ }^{15}$ EIT can estimate the end-expiratory lung volume (EELV) gain during PEEP titration, in addition to the regional distribution of such tidal volume. Thus, simultaneously selecting PEEP level that achieve least overdistention and least alveolar collapse. (Figure 4)

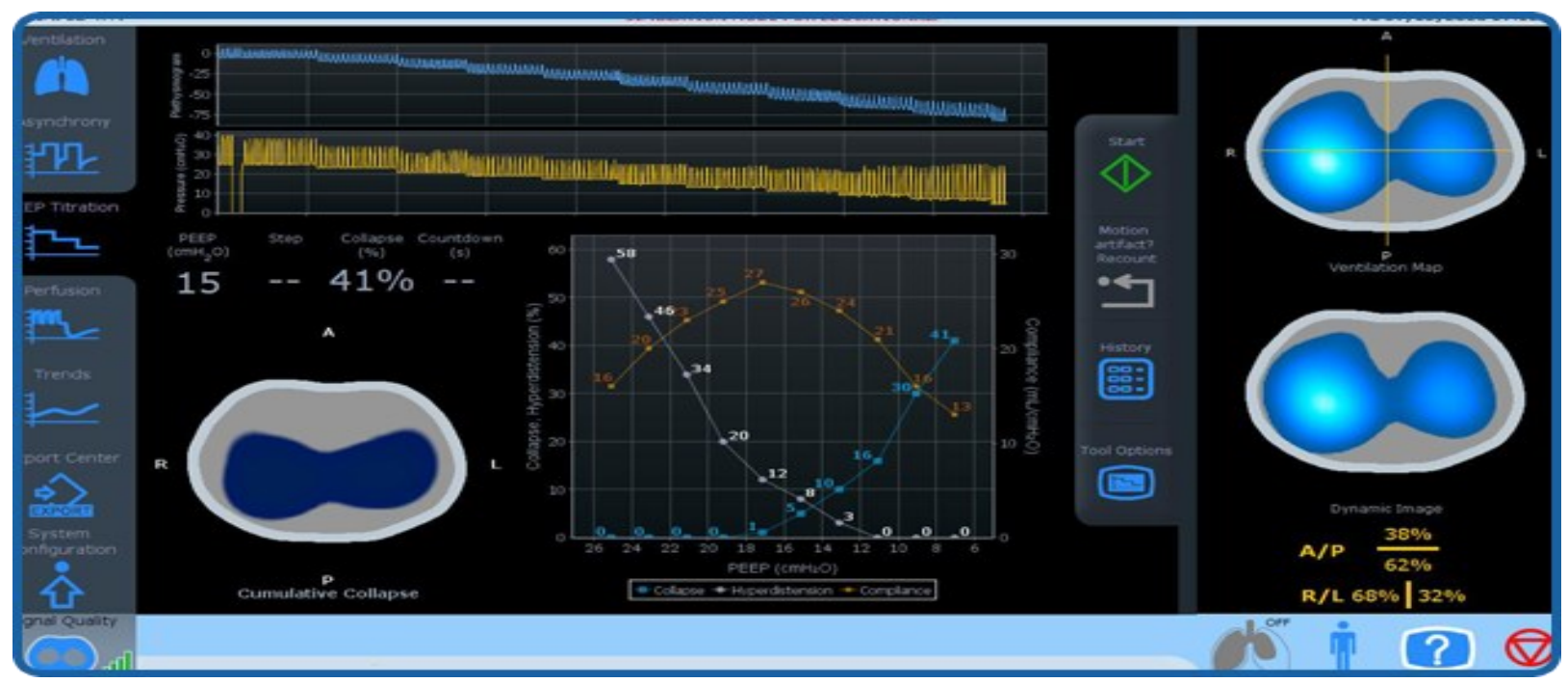

Figure 4: The right image is a ventilation map showing distribution of ventilation between the right and left lung and the anterior and posterior areas. The middle graph showing the effect of decremental PEEP titration on respiratory compliance (orange), collapse percentage (blue) and hyperinflation (white), in this example PEEP of 15 gives the best of all. Courtesy of Timpel reprinted with permission.

\section{Ventilator induced lung injury (VILI)}

By assessing and minimizing areas with overdistention and areas of collapse, EIT has the potential of reducing regions of lung stress and strain and subsequently volutrauma and atelectrauma. ${ }^{16}$

\section{Pulmonary perfusion and Pulmonary embolism}

Assessing differential pulmonary perfusion is possible using EIT. ${ }^{5}$ Coupled with ventilation images, measuring perfusion can assess the Ventilation/Perfusion (V/Q) mismatch. Based on small animal studies comparing EIT to CT scan ${ }^{17}$, and PET scan. ${ }^{18}$ EIT has implications of detecting Pulmonary embolism immediately with no radiation or contrast exposure or moving the patients. 19

\section{Evaluation Pleural effusions/Pneumothoraces}

Studies have evaluated the use of EIT in detection the presence and the effect of pleural effusions (Figure 5). ${ }^{20}$ Additionally, early detection of pneumothoraces using EIT in real time showed high sensitivities and specificities (Figure 6). ${ }^{21}$ 

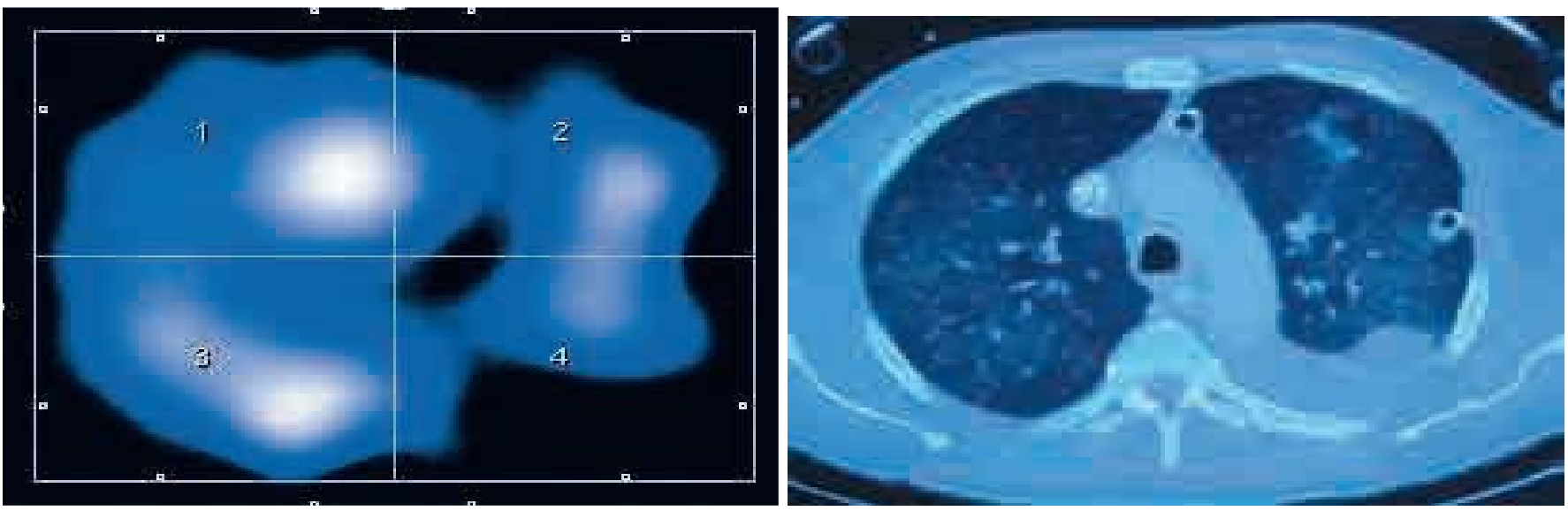

Figure 5: EIT and corresponding CT scan images of a patient with left sided pleural effusions. (C) Drager. Image reprinted with permission.
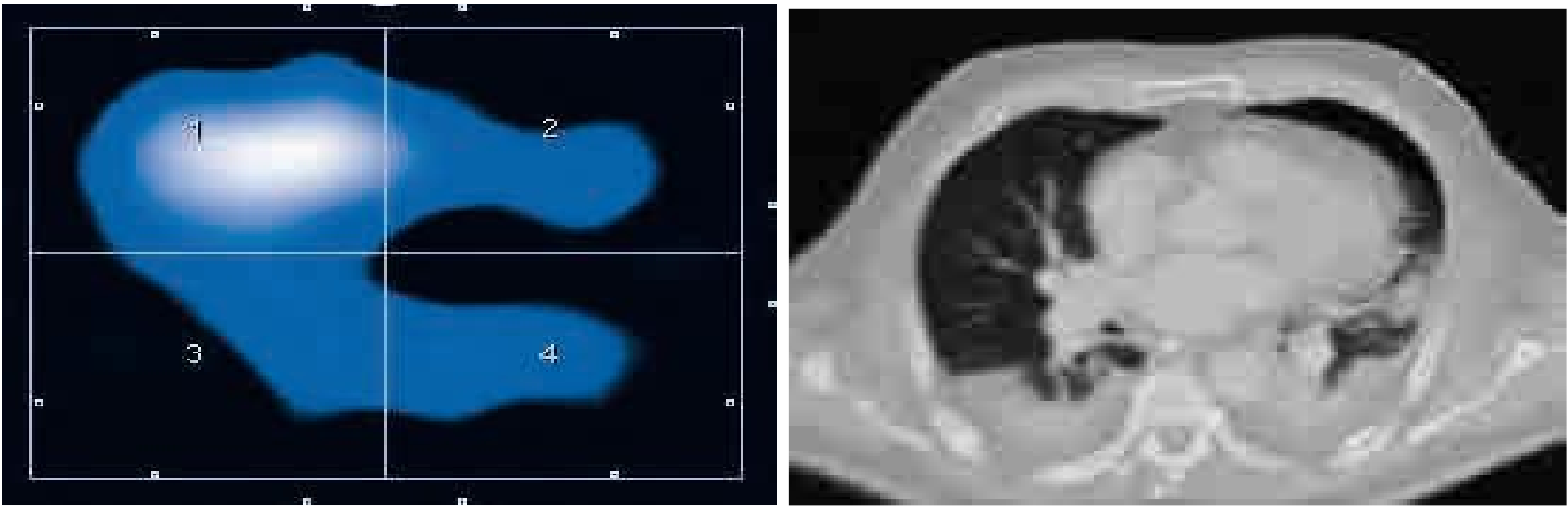

Figure 6: EIT and corresponding CT scan images of a patient with left sided pneumothorax. C Drager. Image reprinted with permission.

\section{Cardiac output}

Fascinatingly, new EIT technology might be able to measure stroke volume and cardiac output ${ }^{22}$ which would be invaluable non-invasive tool for monitoring alongside the lung ventilation and perfusion.

\section{Endotracheal intubation}

EIT may enable incorrect ETT placement (esophageal or right main stem intubation) without further radiological confirmation. ${ }^{23}$

\section{Limitations}

Some limitations of EIT include low resolution in pixels compared to CT scan, the gold standard imaging of the lungs. Morbid obesity (BMI $>50$ ) may result in low quality images. The use of EIT with pacemakers, cardiac defibrillators or during MRI might be relatively contraindicated.
Additionally, given the belts are usually applied circumferentially to the chest, it always gives an axial image of the cross section of the chest but not a cranial or sagittal view. ${ }^{5}$

\section{Future}

Currently, all commercial EIT monitors are standalone monitors, theoretically incorporating this technology and incorporate it in the ventilator might be more beneficial for widespread use.

Expanding the lung areas covered, along with different views might also add a more comprehensive monitoring of the markedly heterogenous lung.

\section{Conclusion}

Though not a new technology, the accelerated advances and research over the last decade had made it possible to have such technology available today as 
a valuable bedside monitoring with multitude of benefits that will revolutionize the way we approach mechanical ventilation.

More well-designed studies and further enhancement in the technology are needed and are forth coming to give us a better understanding of such a technology.

\section{References}

1. Stefan MS, Shieh MS, Pekow PS, et al. Epidemiology and outcomes of acute respiratory failure in the United States, 2001 to 2009: a national survey. J Hosp Med 2013; 8(2):76-82.

2. Qadir N, Mathew R. Imaging of the Critically Ill Patient: Radiology. In: Oropello JM, Pastores SM, Kvetan V. eds. Critical Care. McGraw-Hill; Accessed May 08, 2021.

3. Danish M, Agarwal A, Goyal P, et al. Diagnostic Performance of 6-Point Lung Ultrasound in ICU Patients: A Comparison with Chest X-Ray and CT Thorax. Turk J Anaesthesiol Reanim 2019; 47(4):307-319.

4. Costa, Eduardo, Lima, Raul, Amato, Marcelo. Electrical impedance tomography. Curr Opin Crit Care 2009; 15(1):18-24.

5. Leonhardt S, Lachmann B. Electrical impedance tomography: the holy grail of ventilation and perfusion monitoring? Intensive Care Med 2012; 38(12):1917-1929.

6. Adler A, Amyot R, Guardo R, et al. Monitoring changes in lung air and liquid volumes with electrical impedance tomography. J Appl Physiol 1997; 83:1762-1767.

7. Hinz J, Hahn G, Neumann P, et al. End-expiratory lung impedance change enables bedside monitoring of end-expiratory lung volume change. Intensive Care Med 2003; 29:37-43.

8. Lobo B, Hermosa C, Abella A, Gordo F. Electrical impedance tomography. Ann Transl Med 2018;6(2):26.

9. Gattinoni L, Tonetti T, Quintel M, et al. Regional physiology of ARDS. Crit Care 2017; 21:312.

10. Lehmann S, Leonhardt S, Ngo C, et al. Electrical impedance tomography as possible guidance for individual positioning of patients with multiple lung injury. Clin Respir J 2018; 12(1):68-75.
11. Tomasino S, Sassanelli R, Marescalco C, et al. Electrical Impedance Tomography and Prone Position During Ventilation in COVID-19 Pneumonia: Case Reports and a Brief Literature Review. Semin Cardiothorac Vasc Anesth 2020; 24(4):287-292.

12. Gattinoni L, Caironi P, Cressoni M, et al. Lung recruitment in patients with the acute respiratory distress syndrome. N Engl J Med 2006; 354(17):1775-1786.

13. Hess DR. Recruitment Maneuvers and PEEP Titration. Respir Care 2015; 60(11):1688-1704.

14. Daoud EG, Shimabukuro R. Mechanical ventilation for the non-critical care trained practitioner. Part 1. J Mech Vent 2020; 1(2):39-51.

15. Sahetya SK, Goligher EC, Brower RG. Fifty Years of Research in ARDS. Setting Positive EndExpiratory Pressure in Acute Respiratory Distress Syndrome. Am J Respir Crit Care Med 2017; 195(11):1429-1438.

16. Beitler JR, Malhotra A, Thompson BT. Ventilator-induced Lung Injury. Clin Chest Med 2016; 37(4):633-646.

17. Frerichs I, Hinz J, Herrmann P, et al. Regional lung perfusion as determined by electrical impedance tomography in comparison with electron beam CT imaging. IEEE Trans Med Imaging 2002; 21(6):646652.

18. Bluth T, Kiss T, Kircher M, et al. Measurement of relative lung perfusion with electrical impedance and positron emission tomography: an experimental comparative study in pigs. Br J Anaesth 2019; 123(2):246-254.

19. Nguyen DT, Chik W, Kosobrodov R, et al. Pulmonary embolism detection with electrical impedance tomography. Heart, Lung and Circulation 2013; 22: S192-S193.

20. Arad M, Zlochiver S, Davidson T, et al. The detection of pleural effusion using a parametric EIT technique. Physiol Meas 2009; 30(4):421-428.

21. Costa EL, Chaves CN, Gomes S, et al. Real-time detection of pneumothorax using electrical impedance tomography. Crit Care Med 2008; 36(4):1230-1238. 
22. Hochhausen N, Dohmeier H, Rossaint R, et al. Monitoring of cardiac output and lung ventilation by Electrical Impedance Tomography in a porcine model of acute lung injury. Annu Int Conf IEEE Eng Med Biol Soc 2017; 2017:352-355.

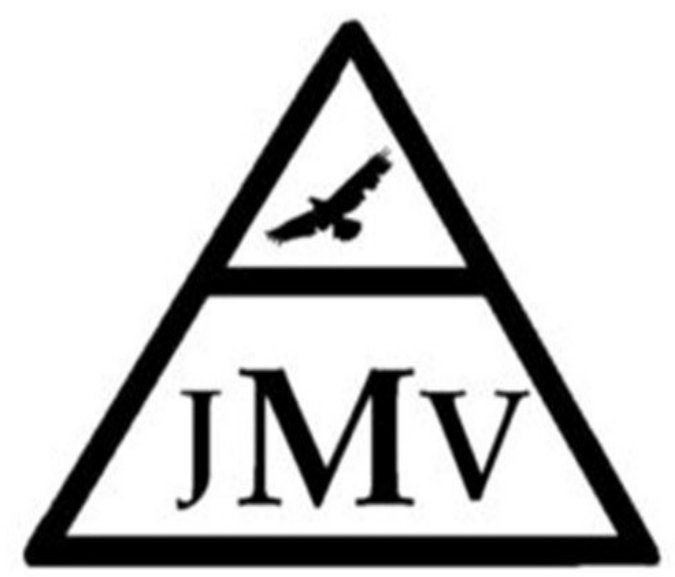

Journal of Mechanical Ventilation

\author{
Submit a manuscript
}

https://www.journalmechanicalventilation .com/submit-a-manuscript/
23. Steinmann D, Engehausen M, Stiller B, et al. Electrical impedance tomography for verification of correct endotracheal tube placement in pediatric patients: a feasibility study. Acta Anaesthesiol Scand 2013; 57(7):881-887.

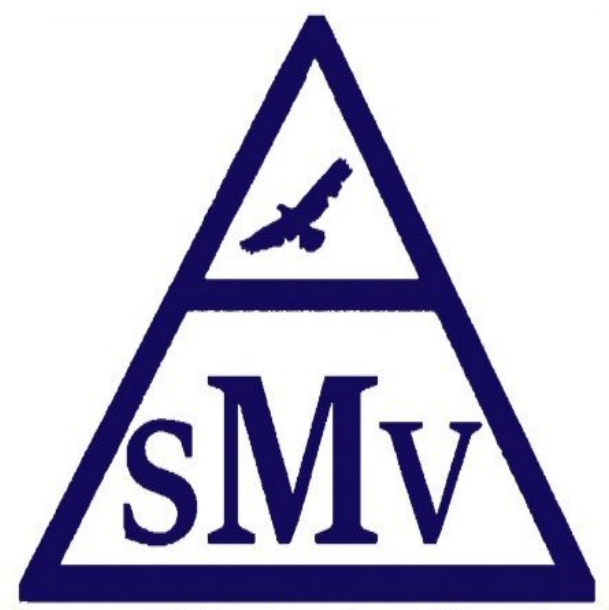

Society of Mechanical Ventilation

Free membership

https://societymechanicalventilation.org /membership/ 